\title{
Efectos socioterritoriales de la mega minería y reacción social: el caso de Minera Alumbrera en la provincia de Catamarca, Argentina ${ }^{1}$
}

\author{
Annie Lamalice ${ }^{2}$ y Juan-Luis Klein ${ }^{3}$
}

\begin{abstract}
RESUMEN
Este artículo aborda la implementación de la minería a gran escala y los efectos socioterritoriales que esta genera a través del estudio de caso de Andalgalá, ciudad argentina ubicada en la zona donde se llevó a cabo el proyecto minero a cielo abierto Bajo la Alumbrera. Se realizaron entrevistas a diversos actores socioeconómicos involucrados con el objetivo, por una parte, de documentar las transformaciones socioterritoriales provocadas en la comunidad local, producto de la intervención minera, y, por otra parte, de analizar la respuesta de la sociedad civil, y en particular el rol jugado por la mujer en el movimiento social de oposición a la megaminería. Se concluye que los beneficios del megaproyecto para la comunidad son débiles y mal redistribuidos, que las desigualdades se han visto acrecentadas en diversas escalas, y se destaca el papel protagónico que ha jugado la mujer en el movimiento social engendrado en la oposición a este proyecto extractivo.
\end{abstract}

Palabras claves: Catamarca, Andalgalá, transformación socioterritorial, movimiento social, extractivismo, genero.

\begin{abstract}
This article addresses the implementation of large-scale mining projects and their social and spatial effects in the Argentinian province of Catamarca. A case study was carried out in the town of Andalgalá, located within the area of operation of the open-pit mining project Bajo la Alumbrera. Interviews were made with various stakeholders affected by the project with the objective to analyze, on one side, different dimensions of socio-territorial transformations provoked by the project, and, on the other side, the civil society-based movement which emerged in reaction to it and namely the role played by women. The article concludes that the benefits of the megaproject are weak and poorly redistributed, that the inequalities have been reinforced at different scales, and that women have been important protagonists of the social movement triggered by the opposition to the project.
\end{abstract}

Key words: Catamarca, Andalgalá, socio-territorial transformation, social movement, extractivism, gender

Este artículo resume los resultados de una tesis de maestría (M.Sc) realizada en el departamento de geografía de la Universidad de Quebec en Montreal por A. Lamalice, bajo la dirección de J.-L. Klein. Ver Lamalice (2014). Los autores agradecen a Wilfredo Angulo y Reina Victoria Vega por su ayuda en la revisión de este texto. Artículo recibido el 28 de diciembre de 2015, aceptado el 27 de mayo de 2016 y corregido el 19 de agosto de 2016

Departamento de Geografía, Universidad de Québec en Montreal (Canadá).

E-mail: annie.lamalice@umontreal.ca

Centre de recherche sur les innovations sociales (CRISES) / Departamento de Geografía, Universidad de Québec en Montreal (Canadá). E-mail: klein.juan-luis@uqam.ca 
El crecimiento de la economía global en las últimas décadas ha requerido de una gran cantidad de energía y materias primas extraídas de los subsuelos (Magrin \& Perrier-Bruslé, 2011). Esta demanda creciente, proveniente principalmente de las potencias económicas emergentes de Asia, sobretodo de China, ha conducido a la proliferación de proyectos de inversión minera de gran envergadura (Gudynas, 2007). El territorio sudamericano, por sus numerosos e importantes recursos mineros, ha resultado ser particularmente atractivo para el gran capital internacional en búsqueda de nuevos yacimientos donde invertir, atracción facilitada por la apertura al libre mercado en varios países de esta región (De Mattos et al., 2005). Como se sabe, a partir de los años 1980, la conversión al neoliberalismo de estos países se inscribe en un contexto de fragilidad marcado primero, por la presencia de dictaduras militares y luego, por procesos de implantación de una democracia debilitada por la incapacidad de los estados de proteger adecuadamente a la economía nacional (Dabène, 2012, Velut, 2009). La convergencia de la demanda internacional en materia de recursos y de las reestructuraciones en la gobernanza de las economías nacionales ha acentuado el papel de América del Sur en la división internacional del trabajo como exportador de recursos naturales (Gudynas, 2007; Svampa 2011; Machado Araoz, 2009a).

Durante la década de los noventa, América Latina fue el territorio que recibió el mayor porcentaje de la inversión de capitales en el sector minero en el mundo. Entre 1990 y el 2001, de las 25 inversiones extranjeras directas (IED) más importantes en este sector, 12 tuvieron lugar en esta área (Bebbington et al., 2008) y entre los diez países con más inversiones en exploración minera, cuatro eran sudamericanos: Chile, Perú, Brasil y Argentina. En la década siguiente, las IED en Argentina se multiplicaron por 10, pasando de 660 millones a 7,35 billones de dólares US. Al mismo tiempo, el número de proyectos mineros paso de 40 a 403 y los puestos de trabajo en este sector crecieron de 98.700 a 258.000 (Secretaria de Minería de la Nación, 2013).

La economía de varios países sudamericanos, particularmente de Argentina, que, a través de la modernización y de la sustitución de las importaciones favorecida por la Comisión económica para América latina y el Caribe (CEPAL), había adquirido las características de un sistema fordista periférico (Lipietz, 1984), se vio modificada como resultado de la implantación de un modelo extractivista (Machado Aráoz, 2009b). Este modelo, que Gorenstein (2015) ha caracterizado como de "reprimarización", se basa en la explotación de los recursos naturales e incluye tanto a las actividades extractivas como tales (minería e hidrocarburos), como al resto de las actividades primarias (la agroindustria por ejemplo). Su lógica consiste en fomentar la monoproducción y la conversión a la producción primaria destinada a la exportación (Svampa, 2011; Lacombe, 2012). En Argentina, la transición hacia el extractivismo afecta en particular al segmento femenino de la población activa. Esto explica, como se verá en nuestro estudio de caso, que la mujer haya jugado un papel protagónico en la oposición a este modelo (Canovas, 2008; Maris, 2009).

En este artículo, analizaremos las mutaciones socioterritoriales provocadas por el desarrollo del megaproyecto minero a través de la lenta transformación de la identidad de la población local, de la disminución de los servicios públicos y de la reestructuración de la economía local. A través de estos temas, también se analizará la situación de las mujeres y su papel en el movimiento social opuesto a la minería. El caso que estudiaremos es el de la implantación del proyecto minero Bajo la Alumbrera, dirigido por Minera Alumbrera, en la provincia de Catamarca, en el Noroeste de Argentina (NOA). En primer lugar, describiremos la región del Noroeste argentino en tanto que región periférica afectada por problemas de pobreza, cuyo desarrollo ha sido desequilibrado por la actividad minera. En esta sección también se describirán los aspectos esenciales de la 
ciudad de Andalgalá. En segundo lugar, presentaremos el marco teórico del estudio, así como los conceptos más importantes. Se abordará el concepto de territorio en el contexto de la globalización, contexto en el cual se emplazará la discusión sobre el modelo extractivista y el tema de la precariedad social y territorial engendrada por la globalización. En tercer lugar, presentaremos el marco metodológico de la investigación realizada, en particular las entrevistas llevadas a cabo en Andalgalá. En cuarto lugar, describiremos en detalle los resultados de estas entrevistas en lo que respecta al conflicto socioterritorial engendrado por el proyecto minero, y haremos énfasis en el rol de la mujer en este conflicto. En quinto lugar, analizaremos las transformaciones socioterritoriales provocadas por la implantación de la gran minería. Insistiremos en el clima conflictivo y en el sentimiento de precariedad generalizado como consecuencia del proyecto minero. En la conclusión, abordaremos la importancia de aportar una respuesta social al modelo impuesto por la globalización, la cual se debe de incluir un componente género importante.

\section{Andalgalá en el Noroeste argentino: una región periférica}

En términos socioeconómicos, la región del NOA es una de las zonas más afectadas por la pobreza en el país. El $40 \%$ de la población que habita este territorio vive con ingresos inferiores al umbral de pobreza. (Natera Rivas, 2005). Con respecto a la provincia de Catamarca ${ }^{4}$, ella presenta una de las tasas de desempleo más altas del país. En algunas áreas de la región, tal como el departamento de Andalgalá, la población desempleada llega a afectar a un tercio de la población en las zonas rurales de la provincia (Veneranda, 2012). En el 2011, el salario medio en la provincia de Catamarca era un $12 \%$ más bajo que el promedio nacional. Ese mismo año, en lo que atañe a la intensidad de las desigualdades sociales, dicha provincia ocupó la séptima posición entre las 23 provincias del país. En lo concerniente al peso de Catamarca en la economía argentina, el producto interno bruto de la provincia representa alrededor del $1 \%$ de la economía nacional y vale resaltar que la minería representa el $70 \%$ del PIB de la economía provincial. En el seno del sector minero, el rubro más importante es el cobre (Dirección Nacional de Relaciones Económicas con las Provincias, 2012).

En plano histórico hay que resaltar que la pequeña minería o minería artesanal en Catamarca remonta a los tiempos del imperio incaico, en la época prehispánica. Sin embargo, esta industria cambió por completo cuando el consorcio formado por Xstrata (Suiza, 50\%), Goldcorp (Canadá, 37,5\%) y Yamana Gold (Canadá, 12,5\%) adquirió una concesión minera en 1994 con el propósito de explotar, por primera vez en Argentina, una mina a cielo abierto. Inicialmente, este megaproyecto minero fue bien recibido por la población. Las promesas de un rápido desarrollo económico y social a través de la creación de miles de puestos de trabajo y de mejora de los servicios generaron entusiasmo en los departamentos concernidos por el proyecto y en la capital provincial (Machado Aráoz, 2009b). Sin embargo, como veremos, el desencanto y la oposición no tardaron en generarse frente a las promesas incumplidas.

\footnotetext{
En Argentina hay 23 provincias, que se dividen en departamentos. Andalgalá es uno de los 16 departamentos de la provincia de Catamarca. La capital de la provincia de Catamarca es San Fernando del Valle de Catamarca, también llamada, en su forma corta, "Catamarca". La provincia de Catamarca posee una superficie de $102.602 \mathrm{~km}^{2}$ y su población actual es de 367.828 habitantes, que representa 0,92\% de la población argentina. La mitad de la población reside en la capital. Los otros 15 departamentos tienen una densidad poblacional de alrededor de $2 \mathrm{~h} / \mathrm{Km}^{2}$ (INDEC, 2010).
} 
Nuestra investigación se realizó principalmente en la ciudad de Andalgalá, un centro urbano de 14.000 habitantes ubicado $250 \mathrm{~km}$ al norte de la capital de la provincia y $40 \mathrm{~km}$ al sur de donde se desarrolla el proyecto minero objeto de nuestro estudio (Figura $N^{\circ} 1$ ). Andalgalá sufre de un fuerte aislamiento geográfico. Una sola carretera asfaltada conecta a la ciudad con Catamarca y se necesitan tres horas y media para recorrer los 177 kilómetros que separan a las dos ciudades. Los demás caminos son de tierra y representan muchos riesgos, sobre todo en el cruce de arroyos y zonas montañosas escarpadas. Las características geográficas de este territorio y el precario desarrollo de su infraestructura vial limitan las posibilidades de desarrollo de vínculos comerciales con otros departamentos y con la provincia vecina de Tucumán, cuya capital se ubica a solo 150 kilómetros de Andalgalá. Así, se puede estipular que el departamento de Andalgalá y la provincia de Catamarca constituyen una región periférica con respecto a los centros que han acumulado los beneficios del intenso desarrollo económico que caracterizó a Argentina en el siglo XX (Bolsi et al., 2010; Coria, 2007; Machado Aráoz, 2009b).

Figura No 1

Ubicación del proyecto minero bajo la alumbrera

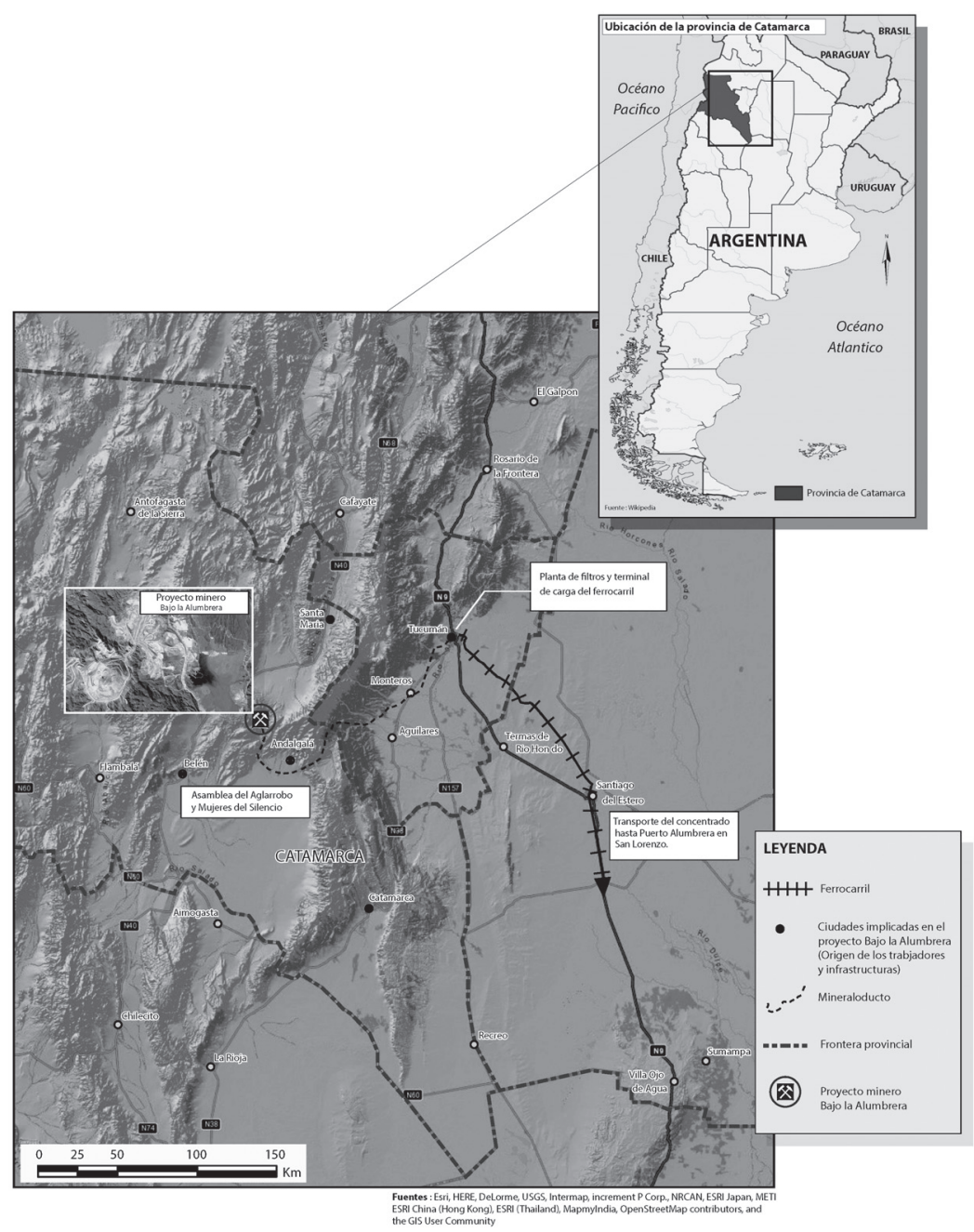

Fuente: Elaboración propia. 


\section{La globalización y el territorio local}

La perspectiva en la cual nos basamos se apoya en una visión social de la geografía según la cual el territorio mediatiza la relación entre la sociedad y el espacio (Klein, 1997; Klein \& Laurin, 1999; Rose \& Gilbert, 2005). Según esta visión, el territorio constituye un espacio delimitado, organizado y ocupado por una colectividad, tanto de manera material (física, productiva, reglamentaria, administrativa, etc.) como inmaterial (códigos sociales, valores, marco cognitivo, percepciones, representaciones, etc.) a través de procesos en los cuales intervienen conflictos y consensos, que se saldan con formas de imposición y de compromiso. Visto de esta manera, el territorio es fuente de lazos sociales pero también engendra percepciones y actitudes diferenciadas e incluso opuestas entre los actores y grupos sociales que lo habitan (Klein, 2008). Esta perspectiva nos permite observar las relaciones que se establecen entre los individuos, los grupos y las sociedades con el espacio, relaciones que determinan la construcción social del territorio y las mutaciones que ella atraviesa (Klein \& Guillaume, 2014).

\section{La configuración socioterritorial provocada por la globalización}

Nuestro análisis de las mutaciones en curso en la pequeña ciudad de Andalgalá se sitúa en el marco de una economía mundial, cuya configuración se transformó completamente en los años ochenta y noventa (Scott, 2001; Sassen, 2007). En el proceso que conduce a esta nueva configuración, las relaciones internas de desarrollo desigual en el seno de los estados se combinan con una economía archipielágica (Veltz, 1996), donde las "regiones que ganan" (Benko \& Lipetz, 1992) emergen en "océanos de pobreza" (Klein et al., 2009), inscribiéndose en dinámicas territoriales diferentes. Las relaciones centro-periferia de nivel nacional, que caracterizaron a la división interregional del trabajo en los diferentes países, pierden su preeminencia y las entidades espaciales que concentran la riqueza (aunque también la pobreza), como las capitales nacionales o las grandes metrópolis, establecen interrelaciones de colaboración y de competencia entre ellas, en un plano internacional. Siguiendo y apoyando esta nueva dinámica, los gobiernos tienden a renunciar a la ambición de desarrollar los territorios más desfavorecidos, concentrando sus recursos en el apoyo a ciudades y regiones competitivas a nivel internacional. En este contexto, las estrategias de reestructuración y de conversión que tanto los Estados como el capital ponen en juego intensifican y aceleran las tensiones y las desigualdades provocadas por la globalización. Los territorios periféricos quedan cada vez más a merced de las inversiones directas internacionales, lo que tiene importantes efectos sociales, económicos y ecológicos sobre su poblacion. El resultado de todo esto es que el territorio nacional pierde importancia en tanto que marco estructurante de relaciones sociales de producción, de regulación y de gobernanza, tomando relieve el papel del territorio local en tanto que sistema de actores a nivel territorial (Klein, 2005; 2008).

Con respecto a este contexto general, los países latinoamericanos presentan sesgos específicos (Gudynas, 2005), donde se hace evidente una despreocupación pronunciada de parte del Estado por los territorios más desposeídos. La debilidad del Estado en lo que respecta a la protección de los derechos ciudadanos en estos territorios contrasta con sus grandes esfuerzos para proteger en los mismos territorios al gran capital extranjero y nacional. Esto intensifica las relaciones conflictivas entre las empresas multinacionales y la ciudadanía (Machado Aráoz, 2009b).

Existen divergencias significativas entre la visión del territorio de las poblaciones enraizadas en el ámbito local, quienes dan prioridad esencialmente al valor de uso, y la visión que tienen de 
éste las empresas multinacionales para quienes el valor del territorio depende de la capacidad que éste procura de generar beneficios y que por lo tanto corresponde al valor de cambio y depende de la especulación. El gran capital le impone a las comunidades locales su concepción del espacio y su visión de la valoración de los atributos territoriales (Harvey, 2010). Por lo tanto, hay una expropiación del valor territorial local, lo que se manifiesta en el plano financiero, económico, ecológico y sociocultural. Este proceso propulsa una visión del territorio de tipo mono valor, una visión que le da prioridad al valor de cambio capitalizado por la actividad extractiva. Machado Aráoz (2009a) utiliza el concepto de alienación socioterritorial para describir la imposición de esta visión.

\section{La globalización y el modelo extractivista}

Es en este marco que se inscribe la aplicación del modelo extractivista en América latina, el cual centra la conflictualidad entre los actores en el tema del medioambiente (Svampa et al., 2009). El concepto de "desarrollo geográfico desigual" desarrollado por Harvey (2010) explica la degradación social y ambiental provocada en general por el capitalismo. Dicho fenómeno, que ha sido intensificado por el neoliberalismo y la globalización, ha estimulado el surgimiento de movimientos sociales que combinan la reivindicación social con la defensa del territorio, en particular en su dimensión medioambientalista. En numerosos territorios afectados por la degradación social y ambiental, provocada por el capital trasnacional se han venido engendrando movimientos socioterritoriales orientados hacia la defensa del medio ambiente (Walter, 2009), los cuales involucran a las comunidades afectadas por los impactos de proyectos extractivos (Orellana, 1999).

Un aspecto importante que debe ser subrayado es que los efectos de los proyectos extractivos tienen repercusiones diferenciadas según el género de las personas afectadas, lo que implica también una reacción social diferenciada según el género (Hartsock, 2008; Leblanc, 2010). Esto nos lleva a plantear que los movimientos sociales de reacción a los proyectos extractivos son de naturaleza territorial multiescalar y multigénero (Svampa et al., 2009).

\section{La precariedad social y territorial}

Uno de los principales efectos del modelo impuesto por la globalización es la precariedad. La precarización se define como una posición de fragilidad, de vulnerabilidad y de incertidumbre frente al futuro (Alpe \& Beitone, 2007). De este modo, la precariedad se opone a la estabilidad y produce exclusión (Alpe \& Beitone, 2007) e incluso desafiliación (Castel, 2008). La precariedad se manifiesta en el nivel social como resultado del debilitamiento del estado social y de la capacidad (o de la voluntad) protectora del estado (Mingione, 2016), lo que deja a una parte de la ciudadanía con acceso limitado al ejercicio de sus derechos en tanto que ciudadanos (Castel, 2008). También se manifiesta en el campo productivo, en lo que respecta a los cambios en el mercado del trabajo; los cuales se saldan con la disminución de la protección de numerosas capas de trabajadores (Soussi \& Beaudet, 2014). Este concepto de precariedad permite de abordar los impactos territoriales desiguales inducidos por las inversiones extractivas, y también permite distinguir dichos impactos en relación con el género puesto que la precariedad afecta de manera diferente al hombre y a la mujer (Chiasson-LeBel, 2006). 


\section{Metodología del estudio}

La investigación que sustenta este artículo se basa en el método del estudio de caso. Se eligió este método porque permite analizar un fenómeno a profundidad y en detalle. Se combinaron diferentes técnicas para la recolección de los datos necesarios para la comprensión del objeto de estudio: el análisis de documentos, la observación directa y la entrevista semiestructurada. El artículo hace énfasis en los resultados de las entrevistas, las cuales fueron realizadas en la provincia de Catamarca entre junio y octubre del año 2013. El uso de esta técnica nos ha permitido comprender y explicar los efectos y las reacciones con respecto al auge de la minería argentina. El caso objeto de estudio, es decir el proyecto minero "Bajo la Alumbrera" implantado por el consorcio Minera Alumbrera, es sumamente instructivo porque fue considerado como uno de los grandes éxitos de las políticas extractivas iniciadas por el presidente Carlos Menem en la década de los noventa. Por otra parte, la implantación de este proyecto ha provocado una fuerte reacción social, habiéndose realizado, a fines de los noventa, una asamblea ciudadana que adoptó el nombre de "Asamblea del Algarrobo". Además, el hecho de que este proyecto se encuentre en la zona del noroeste es también significativo puesto que es allí donde se concentra la mayor parte de la minería argentina. Por último, el hecho que el proyecto se esté acercando a las dos décadas de funcionamiento permite observar los cambios generados en el territorio. Estas razones hacen del proyecto "Bajo la Alumbrera" un caso icónico.

En lo que respecta al trabajo de campo como tal, para las entrevistas hemos optado por un muestreo no probabilístico. Con la ayuda de informantes claves de la colectividad, hemos construido una muestra utilizando el método de la bola de nieve. Este método nos permitió un contacto directo con diferentes grupos de actores. La muestra se compuso de 26 personas posicionadas bien sea a favor o bien en contra de la industria minera en la provincia de Catamarca habitantes del departamento de Andalgalá donde se ubica el centro urbano más cercano al proyecto minero.

Las entrevistas fueron realizadas con el apoyo de una guía temática. Sobre la base de esta guía, se interrogó de manera específica en relación con su campo de acción a las personas entrevistadas. Además, nos hemos servido de algunas intervenciones de actores del sector minero con respecto al tema de las mujeres en la industria minera. Estas intervenciones tuvieron lugar en la ciudad de San Fernando del Valle de Catamarca el 26 de septiembre de 2013. También se emplearon fragmentos de discursos del Ministro de Producción y Desarrollo de la Provincia de Catamarca, Angel Mercado, y de la Secretaria Corporativa de la Asociación Obrera Minera Argentina (AOMA), María Elena Isasmendi.

\section{El conflicto socioterritorial en Andalgalá}

Andalgalá es una ciudad con casi 14.000 habitantes. En esta ciudad es donde han tenido lugar los principales debates y conflictos acerca del proyecto Bajo la Alumbrera. Desde el principio, la población se dividió en dos polos: los "promineros" y los "antimineros". Esta polarización tuvo efectos importantes en la vida comunitaria de esta ciudad puesto que dividió a los vecinos, a los amigos y a las familias, lo que se tradujo en una importante deterioración de la cohesión social en el seno de la ciudad. En este sentido, podemos citar lo referido por una de las entrevistadas: 
"Yo cumplí cincuenta años el año pasado, mi esposo me regalo una fiestita así chica con treinta, cuarenta invitados, amigos y familia. Y bueno tenemos el parque ahí y había puesto dos mesas con seis sillas cada una, y era notorio cuando llegaba la familia y algunos amigos que miraban así y a dónde se iban. Teníamos una mesa acá en favor de la mina, los otros de allá que son los antimineros. ¡Mira aquellos se han juntado! Pero era una reunión de familia, porque era familia más unos cuantos amigos muy muy cercanos, nada más. Y hasta en eso se notaba". (Entrevista 20, con una profesora).

Esta polarización explica porque cuando pedimos a los actores que se pronuncien sobre los efectos causados por la implementación del megaproyecto minero en su departamento, las respuestas varían dependiendo de si ellos se identifican como promineros o antimineros. Esta división, a pesar de que existe desde el inicio de la década 2000, se ha intensificado bastante después del 2010. El 15 de febrero de ese mismo año, cuando la empresa estaba transportando la maquinaria pesada al Aconquija (la montaña que domina Andalgalá) para un nuevo proyecto llamado "Agua Rica", los principales actores antimineros organizaron un bloqueo con el objeto de impedir la circulación de los vehículos relacionados con la industria minera. La policía y el ejército fueron desplegados y la represión fue violenta. La represión del 15 de febrero intensifico la confrontación entre los que apoyan y los que se oponen a la megaminería. Por eso, este tipo de eventos, que antes eran raros en el departamento de Andalgalá, se han ido volviendo recurrentes.

\section{El sentimiento de pertenencia y la identidad}

Los Andalgalenses son muy apegados a su departamento y a su provincia. Sin embargo, el sentimiento de pertenencia se ha reducido entre los jóvenes, lo que los lleva a migrar a los grandes centros urbanos o a otros países, como nos lo dice un entrevistado:

“Otro problema grave es el éxodo de la gente joven de nuestro departamento hacia los grandes centros. (...) En Andalgalá entre los niveles, la escuela secundaria y terciaria, ingresan todos los años entre 350 y 450 alumnos. Chicos que quieren trabajar pero no tienen posibilidad de hacerlo. Entonces qué hacen, tienen que migrar o tienen que ir a golpear a la puerta del intendente o un concejal o un diputado o un gobernador que le dé un cargo en la administración pública generando más déficit..." (Entrevista 21, con un empresario).

La decisión de dejar Andalgalá se explica por la falta de trabajo y de posibilidades de estudio, pero también por el tenso clima social que reina en Andalgalá y del cual los jóvenes quieren escapar. Se ve aquí claramente que la percepción del lugar ha ido adquiriendo rasgos negativos. Esta situación fue reportada en diferentes entrevistas, por ejemplo la realizada con un activista que tiene cuatro hijos, de los cuales tres han optado por vivir en diferentes ciudades, lejos de Andalgalá, quienes afirman "no querer vivir el mismo infierno que sus padres" (Entrevista 12, con un activista).

\section{El declive de los servicios}

Como en todas las regiones periféricas, en Andalgalá los servicios de salud y de educación son insuficientes. Sin embargo, conociendo el auge de la industria minera en la zona, se esperaban mejoras en la calidad de los servicios, sobre todo si se tiene en cuenta que el argumento principal para promover el proyecto minero había sido el efecto positivo que la riqueza producida por la industria minera debía tener sobre la calidad de vida de la comunidad. Pero ha sido todo 
lo contrario. Examinemos, a título de ejemplo, la percepción de los entrevistados acerca de los servicios de salud y de educación.

Con respecto al sistema de salud, recordemos que en Argentina, este funciona a dos niveles. Por un lado, se encuentra el sistema público de acceso gratuito para todos $y$, del otro lado, el sistema privado, que beneficia a los individuos que pueden pagarlo y a los trabajadores protegidos por seguros. Evidentemente, el sistema privado es de acceso limitado. Pero lo que surge principalmente de las entrevistas es que el sistema público es cada vez menos accesible. Un entrevistado que trabaja en el sistema médico de Andalgalá afirma que la salud está afectada por dos problemas. En primer lugar, refiere que el personal médico y los recursos técnicos son escasos. En segundo lugar, que el servicio disponible en Andalgalá es muy básico. Los pacientes deben dirigirse a la ciudad de Catamarca por cualquier problema que exija competencias más especializadas:

"Políticamente, el sistema de salud no es algo que interese. (...) Entonces alguien que necesita una tomografía tiene que hacer $240 \mathrm{~km}$ para ir a Catamarca y hay lugares que son mucho más alejados y con caminos difíciles de llegar. Por ejemplo te doy una idea: acá yo luché para tener una diálisis renal, y la gente iba, salía a las dos de la mañana en micro, y volvió a la 9 de la noche. Y si alguna vez viste alguien que sale de diálisis: salen mal" (Entrevista 1 , con trabajador del sistema médico).

Durante muchos años se ha luchado por obtener algunos equipos médicos, pero la dotación sigue viéndose como muy insuficiente. El hecho de que una buena parte de los pacientes sean enviados a Catamarca, a $240 \mathrm{kms}$ de distancia, además de constituir un riesgo para los pacientes, reduce la accesibilidad a los servicios de salud, puesto que si la atención en los hospitales públicos es gratis, los costos incurridos para el transporte no lo son. Y esto, sin contar el costo adicional para la familia del paciente que en general lo acompaña.

Y aún, los equipos con los cuales se cuenta, no siempre pueden ser aprovechados con un máximo rendimiento. Tal como lo afirma una entrevistada, no basta con tener el equipo necesario, también se necesitan los accesorios y el personal habilitado para usarlo e interpretar los resultados.

"El tema del acceso a los servicios públicos es limitado. Tienes un hospital que tiene aparatos nuevos pero no tiene personal que lo maneje. (...) Acá tienes un mamógrafo nuevo y todavía no tienes la tinta para imprimir los informes... y es ridículo en el fondo porque puedes tener la infraestructura pero no tienes por parte del gobierno la gana de decir: "Che pongamos un especialista que atienda en Andalgalá (...) y haga mamografía" (Entrevista 16, con una empleada).

Con respecto a la educación, la situación es similar. La educación en Argentina es pública, gratuita y obligatoria hasta el nivel secundario. Las entrevistas muestran que el sistema escolar es satisfactorio en lo que respecta a los niveles primario y secundario. Pero, cuando se trata de la educación postsecundaria, surgen diversos problemas. En primer lugar, hay que recordar que este nivel incluye la formación técnica y la formación universitaria. En Andalgalá, hay un Instituto de Estudios Superiores, pero no hay ninguna universidad. Los jóvenes que desean continuar su educación después de la escuela secundaria deben conformarse con la oferta del instituto o 
trasladarse a una ciudad donde haya una universidad. Esta última opción solo es posible para los jóvenes de familias adineradas puesto que, aunque la universidad sea gratuita, los estudiantes deben alojarse, alimentarse y desplazarse por su propia cuenta.

En cuanto a los programas que se ofrecen en el instituto de Andalgalá, las posibilidades son limitadas. Únicamente se ofrecen programas en educación. La única opción es la de convertirse en un maestro a nivel de la escuela primaria o secundaria. Y como la oferta de formación ha sido prácticamente la misma desde la fundación del instituto en 1983, esto ha conducido a una superabundancia de maestros. Los graduados que no pueden encontrar un empleo se ven obligados a dejar Andalgalá o a efectuar un trabajo que no tenga relación con sus estudios. Sin embargo, esto tampoco resulta fácil ya que la tasa de desempleo es alta en la provincia. Evidentemente esto se convierte en un factor que estimula el éxodo entre los jóvenes.

\section{Restructuración económica}

En términos de impacto económico, dependiendo de su posición ideológica, las apreciaciones de los entrevistados son divergentes en cuanto a los efectos de la industria minera. En la entrevista, se abordaron tres aspectos de la transformación económica de la región: el empleo en la industria minera, la distribución de los beneficios y los cambios en otros sectores económicos de la ciudad.

Con respecto al empleo, durante la fase de construcción de la infraestructura del proyecto Bajo la Alumbrera, entre 1995 y 1997, se crearon cerca de 4.000 puestos de trabajo. Sin embargo, luego del término de la construcción, el número de trabajadores en la provincia y en Andalgalá disminuyó abruptamente (Cuadro No 1).

\section{Cuadro No 1}

Empleos en la provincia de Catamarca por Departamento. Proyecto "Bajo alumbrera", 2012

\begin{tabular}{|l|r|r|r|}
\hline \multicolumn{1}{|c|}{ Departamentos } & \multicolumn{3}{|c|}{ Empleos } \\
\hline & Hombres & Mujeres & \multicolumn{2}{|c|}{ Total } \\
\hline Andalgalá & 110 & 12 & 122 \\
\hline Belén & 180 & 14 & 194 \\
\hline Santa María & 150 & 14 & 164 \\
\hline Otros & 175 & 19 & 194 \\
\hline Provincia de Catamarca & 615 & 59 & 674 \\
\hline
\end{tabular}

Fuente: Minera Alumbrera, 2012.

En lo que respecta al trabajo de la mujer en la industria minera subterránea en Catamarca, hay que decir que, históricamente, este tipo de trabajo estaba reservado a los hombres. Las mujeres no tenían derecho a entrar en los túneles debido a una creencia local: "la Madre Tierra se volvería celosa", lo que daría lugar a accidentes en la mina. Las cosas han cambiado lentamente en las últimas décadas y las mujeres han logrado ocupar el $8 \%$ de los empleos en el proyecto "Bajo la Alumbrera" (Minera Alumbrera, 2012). Sus funciones son variadas, salvo que no tienen acceso a puestos de dirección, como nos lo confirma una persona entrevistada: 
"Hay ciertos roles que no los pueden cumplir las mujeres. Como nunca he visto una mujer de gerente. Llegan hasta puestos medios. No sé si será una situación de machismo o una situación porque... no sé... porque quizás también nos condicione el hecho que tenemos... que nos casamos, que somos mamas, tenemos niños que se enferman o que tenemos que ir a la escuela. Quizás eso también nos limita a nosotras" (Entrevista 17, con una empleada).

Este extracto recuerda que el papel tradicional de la mujer en la región está muy ligado a la familia. Un administrador en el ámbito de la salud confirma esta apreciación cuando habla del papel de la mujer en la industria minera: "la mujer sigue siendo la que ordena el hogar. [...] nosotros ayudamos todo lo que quieras pero los hijos siguen estando al cargo de la madre" (Entrevista 1).

Cuando se le pregunta lo que significa para una mujer el hecho de trabajar en la mina, una trabajadora responde así:

"Un desafío impresionante. Porque de un lado vos sos una profesional pero por el otro sos madre, sos esposa y vos tenés que conciliar esas dos partes. Y es muy difícil. Para la mujer sí. Porque todas las mujeres sentimos que tenemos culpa de decir bueno me tengo que ir a la mina 7 días y te tenés que ir porque tu trabajo lo necesita. Pero bueno mientras esto pasa estás pensando en tus hijos, en tu casa, si se habrán acostado bien, si fuiste al acto de la escuela, etc." (Entrevista 16, con una empleada).

El trabajo en la mina está organizado de siguiente manera: el trabajador pasa de siete a diez días en la mina y luego tiene un periodo equivalente de descanso en el lugar de residencia ${ }^{5}$. Esto representa un problema mayor para la organización familiar, especialmente para las mujeres que se consideran responsables del buen funcionamiento de sus hogares y sus familias.

El descontento de la población con la distribución de los beneficios del proyecto "Bajo la Alumbrera" es generalizado y se observa en varios niveles. En primer lugar, a nivel macroeconómico. En este plano, muchos de los entrevistados criticaron el hecho de que la industria se lleva de la provincia un metal de gran valor sin dejar prácticamente nada en ella. Por una parte, las críticas apuntan a la ley 24.196, señalándosele como la fuente del desequilibrio en la distribución de los beneficios económicos de la actividad minera. Además, se señala que la falta de coordinación entre los diferentes niveles de gobierno y los desacuerdos entre ellos han afectado a la redistribución de los beneficios mineros en la comunidad.

Los comerciantes entrevistados critican unánimemente el hecho de que los beneficios económicos se inviertan en otros lugares y no en Andalgalá. Señalan que la provincia de Tucumán, que cuenta con las oficinas de Minera Alumbrera y con una fábrica, es el principal beneficiario de la minería. Hay que decir que en 1994, la compañía había prometido la instalación de un centro de operaciones en Andalgalá. Sin embargo, este centro fue construido en la provincia de Tucumán. También se dice que los otros departamentos de la provincia de Catamarca, especialmente Santa María y Belén, tienen ventajas económicas por el mayor número de puestos de trabajo que tienen en el proyecto "Bajo la Alumbrera". Vale la pena resaltar que las cifras aportadas por Minera Alum- 
brera (Cuadro No 1) confirman la percepción de la comunidad, que sostiene que Andalgalá cuenta con una proporción menor de empleados en la minera que los otros departamentos.

En cuanto al nivel microeconómico, las familias que cuentan con miembros empleados en el proyecto minero están favorecidas económicamente. Sin embargo, los puestos de trabajo disponibles son limitados. Un administrador sostiene que la empresa cuenta con una base de datos de más de 15.000 solicitudes recibidas de personas interesadas en trabajar en el desarrollo minero (Entrevista 16, con una empleada).

Desde la implantación del proyecto "Bajo la Alumbrera", la economía de la provincia, sobre todo en los departamentos de Andalgalá, Belén y Santa María, se ha concentrado en la actividad minera, relegando el resto de las actividades económicas a un segundo plano. Para los defensores de la minería, el desarrollo intensivo de la megaminería es la única solución para el desarrollo de la región. Por ejemplo, sostienen que las condiciones geográficas de la provincia no permiten el desarrollo de actividades agrícolas a gran escala. Según ellos, la minería sería la opción natural para los catamarqueños por lo cual el resto de los sectores económicos solo podría tener un papel accesorio.

"Catamarca tiene $100.000 \mathrm{~km}^{2}$, el $80 \%$ es montañas, el resto de la zona es más o menos plana: valle fértil y muchas zonas desérticas como tenemos acá... Entonces uno piensa competir con la pampa húmeda por ganadería o agricultura: no tiene sentido, no tiene ningún sentido. Nosotros tenemos un clima continental, semidesértico con lluvias entre diciembre y marzo y el resto es casi sin más lluvia, con mucho calor, mucho viento, nieve en alguna oportunidad, entonces dedicarse a la agroganadería no va a hacer lo más acertado porque las condiciones que necesitan los grandes sembradíos o mantener una hacienda de buena calidad de carne es difícil en esas condiciones, las condiciones geográficas de Catamarca" (Entrevista 4, con un ingeniero de minas).

Para quienes se oponen a la megaminería, esta especialización crea precariedad. Según ellos, es importante también apoyar a otros sectores, como la industria agrícola, la artesanía y el turismo. Éstos insisten en la necesidad de identificar opciones para adaptar la economía cuando el proyecto "Bajo la Alumbrera" Ilegue a su término. El sector agrícola ha declinado en las últimas décadas. Los entrevistados más ancianos recuerdan con nostalgia un período más próspero, durante el cual la producción agrícola y vitivinícola constituían partes importantes de la economía local. El propietario de una planta procesadora de alimentos agrícolas se refiere a los cambios que han afectado a la economía local:

"Andalgalá antes tenía una superficie cultivable de 1000 hectáreas, hoy tiene 200. Andalgalá antes tenía un caudal de agua suficiente para esas 1000 hectáreas y más. Hoy se ha visto reducido el caudal de agua. ¿Por qué? Porque las políticas dirigidas de los estados nacional y provincial fueron destinadas a crear lo que yo le llamo una máquina electoral. (...) Cerraron bodegas, cerraron fábricas como esta porque no había cantidad de producción, no vino nadie a instalarse acá en Andalgalá a decir yo pongo una industria, yo la puse porque soy de acá y que quiero a mi pueblo y porque cuando yo la puse en la década del 80 todo lo que envasábamos lo sacábamos de acá. Hoy, el $90 \%$ lo tengo que traer de afuera, iel 90\%! Para darte un cálculo exacto, yo envaso por año un millón de kilos de tomates y aquí en Andalgalá llego a cien mil kilos. Tengo que traer 900 mil kilos de afuera" (Entrevista 21 , con un empresario). 
Este empresario acusa el gobierno de su falta de apoyo a la industria alimentaria, mientras que la industria minera goza de privilegios importantes en el plano económico y fiscal. Muchos entrevistados esgrimen que la agricultura y la viticultura serían útiles para salvar la economía de la región. Se basan en la historia de Andalgalá, que fue un gran productor de frutas y verduras, así como de vinos que se distribuían en todo el país. Un entrevistado recuerda ese período:

"Aquí, de Andalgalá salían camiones a Tucumán. Y de aquí llevábamos la fruta, llevábamos la verdura, eso lo llevábamos a Tucumán. Y de ahí volvían vacíos los camiones. Porque aquí teníamos todo. Ahora se van vacíos y vienen llenos con la verdura, con la fruta, todo al revés..." (Entrevista 6, con un activista local).

Un elemento esencial invocado para explicar la decadencia de la industria agrícola, es el hecho de que la minería compite con la agricultura por el agua. Según los antimineros, consumiendo cantidades fenomenales de agua y contaminándola, la industria minera priva a la agricultura y a la ganadería de este recurso vital (Entrevista 8, con un residente antiminero).

En cuanto a los actores del sector turístico, estos revelaron que hay muchos proyectos, pero que faltan los recursos para realizarlos. La región tiene varios elementos atractivos para atraer a los turistas (paisajes, montañas, sitios arqueológicos), pero una vez más, la falta de una red vial adecuada se apunta como factor agravante. Así como el turismo, el sector de la artesanía sufre de falta de recursos para promover las telas y joyas producidas en la zona. Es con orgullo que los habitantes hablan del poncho tradicional tejido en Catamarca que se ofreció al Papa Francisco durante la visita de la presidenta Cristina Fernández de Kirchner en el Vaticano en 2013.

La agricultura, la viticultura y la artesanía son los sectores económicos tradicionales con los que se identifican los Andalgalenses. La industria minera también forma parte de la historia económica de la región desde más de un milenio. Por esta razón, la Asamblea no se opone a la minería en su forma tradicional subterránea. Lo que se cuestiona es el modelo extractivista, a cielo abierto y la decisión de hacer de la actividad minera a gran escala la principal actividad económica de la provincia. Esto no ha permitido llegar a un verdadero desarrollo socioeconómico para la población catamarqueña, lo que explica este ciudadano:

"En muchos casos empeoró la situación. Porque a la gente no solo que no le trajo beneficios en términos laborales, en términos económicos sino que también se descuidaron una cantidad de cosas que eran muy arraigadas en la costumbre de la gente de este pueblo, que era el apego a la tierra por el tema de la agricultura, la ganadería, todo eso se fue abandonando. Y eso generó mayor desocupación, mayores necesidades para la gente" (Entrevista 5, con un activista).

\section{El papel protagónico de la mujer}

Las mujeres se organizan en movimientos feministas latinoamericanos, pero también juegan un papel especialmente importante en los movimientos de defensa del medioambiente. En este sentido, es interesante observar que la participación de las mujeres en los movimientos sociales que presentan un componente ambiental es superior a su participación en otros movimientos, como el movimiento obrero, por ejemplo (Martínez Alier, 2006). Están particularmente presentes en los movimientos socioambientales en Argentina, así como en toda América Latina, especial- 
mente en las asambleas de ciudadanos que se oponen a los megaproyectos extractivos (Comelli, 2010).

Como se ha dicho, desde la implementación del proyecto "Bajo la Alumbrera" se observa una división social entre los promineros y los antimineros. Entre los antimineros, las mujeres de Andalgalá juegan un papel protagónico. Diversas opiniones fueron expuestas para explicar el papel de las mujeres en este movimiento social. Algunas entrevistadas dijeron, por ejemplo, que se involucran en la lucha por obtener más poder, el cual ha sido tradicionalmente masculino (Entrevista 13, con una empresaria).

En la mayoría de las entrevistas, con relación al papel que tiene la mujer en el movimiento, el término más utilizado es "coraje". En el movimiento socioambiental de Andalgalá, las mujeres se consideran más valientes que los hombres y más capaces de defender lo que les importa. La lucha por la conservación del medio ambiente también se asocia con la función protectora de las mujeres. Las activistas hablan de la importancia de su papel como militantes y como madres, es decir, la combinación de los dos papeles, para la defensa de la vida:

"Defender a la tierra, defender la biodiversidad, obviamente pone la madre, la mujer con su posibilidad de dar la luz en un lugar muy especial, ese paralelo entre mujer y madre tierra se ha elevado mucho últimamente. Entonces bueno, creo que en este aspecto hay un empoderamiento de la mujer, a través de la participación política y de la reconceptualización de ella en su rol social" (Entrevista 8, con una militante de la Asamblea)

La participación de las mujeres en las diferentes acciones colectivas en reacción al proyecto también hizo que ellas fuesen las más penalizadas. A lo largo de este conflicto social, ellas son las que más se han enfrentado con la policía y, por lo tanto, las que más han sufrido los efectos de la judicialización. El enfrentamiento entre las mujeres y la policía, es un hecho corroborado a partir de las estadísticas judiciales, donde sale a relucir que dos tercios de las causas judiciales intentadas lo son contra mujeres. Frente a esta situación, un grupo de mujeres activistas decidió fundar el grupo de "Mujeres del Silencio".

Comenzaron a marchar por las calles de Andalgalá todas las semanas, en silencio, para simbolizar la mordaza impuesta por el gobierno que les impide manifestar contra la industria minera. Su ronda (Figura $\mathrm{N}^{\circ} 2$ ) incluye diferentes lugares, entre ellos las oficinas de la empresa, el ayuntamiento, las oficinas del Ministerio de Justicia y del Ministerio de Economía y la estación de policía. He aquí la prueba de que en el fuerte movimiento social que tiene lugar en Andalgalá, las mujeres son importantes. Una militante del organismo "Mujeres del Silencio" afirma que el carácter valiente de las mujeres se explica por la cultura de la región del NOA:

“Decimos acá que las mujeres somos más valientes, somos muy osadas las mujeres acá. No tenemos temores o podemos llegar a tener temores pero si hay una amenaza, sacamos las uñas. Es algo muy notable: las mujeres de nuestra zona, esa cosa que no tienen temor a la represión, a los golpes, las mujeres salimos" (Entrevista 9, con una militante de la Asamblea).

El grupo de las "Mujeres del Silencio" se convirtió en un símbolo de la lucha de Andalgalá y de la afirmación del papel protagónico que las mujeres ocupan en el movimiento social. Las mujeres 
que participan en la Asamblea del Algarrobo y en el grupo las "Mujeres del Silencio" consideran que la lucha contra los megaproyectos mineros es coherente con su rol social. Según las entrevistadas, éste consiste en proteger el bien común. Por ello es que se sienten responsables de oponerse a un modelo extractivo, que consideran perjudicial para su territorio. La lucha que libran las mujeres en Andalgalá contra los impactos de los megaproyectos mineros asociados al capital trasnacional sobre el medioambiente y la calidad de vida de la población en el ámbito local da testimonio de la aspiración de la mujer por obtener el reconocimiento merecido por su participación en los movimientos sociales (Cánovas, 2008).

Figura $\mathrm{N}^{\circ} 2$

Acciones de la Asamblea del Algarrobo y de las Mujeres del Silencio en Andalgalá



Fuente: Elaboración propia.

\section{Las transformaciones socioterritoriales provocadas por el proyecto minero}

A través de nuestro estudio de caso, nos hemos interrogado acerca de los efectos de los grandes proyectos mineros en las comunidades locales. A la luz de los resultados presentados 
anteriormente, en los siguientes párrafos, discutiremos sobre las principales transformaciones observadas en el seno de la comunidad analizada.

\section{Instauración de un clima social conflictivo}

La vida ha cambiado mucho en Andalgalá a lo largo de los últimos veinte años, pero esto no se debe al desarrollo económico fulgurante que se anunció durante los noventa, cuando se produjo la instalación del proyecto "Bajo la Alumbrera". Las transformaciones que los entrevistados identifican son de carácter negativo y la mayoría se sitúan a nivel social. En general, se puede considerar que el tema de la minería ha polarizado a los residentes de la pequeña ciudad, rompiéndose así la cohesión social que existía antes de la llegada de Minera Alumbrera.

En primer lugar, se observa el deterioro de los lazos sociales, lo cual ha conllevado a la división de las familias y los barrios. Los actores sociales entrevistados afirman que han surgido nuevos problemas sociales como consecuencia de la instalación del proyecto minero. Los ejemplos más citados son el aumento en el consumo de drogas y la prostitución. Un entrevistado, residente de Andalgalá, confirma esta visión negativa de las transformaciones sociales acaecidas en los últimos años:

“Acá llegó lo malo de la globalización, lo malo... la disolución de los valores, el nuevo modelo de familia que no presenta modelo ni paradigma, los chicos... hay mucha droga, mucho alcohol. A los adolescentes de trece, catorce años ya uno los ve alcoholizados..." (Entrevista 21, con un empresario).

Esta cita ilustra el desencanto de la población de Andalgalá con respecto a las transformaciones impulsadas por el desarrollo minero. El anterior punto de vista, donde se afirma que la región recibió solo los aspectos negativos de la globalización, que proviene de un actor entrevistado que pertenece a la comunidad empresarial hace eco de un sentimiento generalizado que resultó ser una suerte de denominador común en el discurso de los entrevistados. Dicho sentimiento pone de manifiesto una sensación concreta de la población local: la distribución desigual de los impactos sociales causados por los grandes proyectos mineros. Al nivel de la región, las relaciones sociales también han empeorado. De hecho, se ha desarrollado la pugna entre cada uno de los centros urbanos por tratar de concentrar el máximo de beneficios económicos. Se ha introducido a escala regional, la competencia entre las ciudades que se observa también a escala nacional e internacional.

En segundo lugar, se observan cambios en la evolución del sentimiento de pertenencia de la población hacia su territorio. El sentimiento identitario se ha mantenido en la población adulta, pero ha mermado considerablemente en la población joven. El clima social conflictivo que se ha instalado en las dos últimas décadas en Andalgalá contribuye al éxodo de los jóvenes. Muy pocos quieren adoptar este estilo de vida que implica una lucha constante por el desarrollo social en un clima de conflicto. De hecho, las dos posiciones discursivas que se enfrentan sobre el desarrollo de la minería, los pro-minería y los anti-minería, provocan muchos comportamientos sociales violentos en Andalgalá generalizándose así un clima de inseguridad, el cual se intensifica debido a la falta de protección de las autoridades:

"A mí por ejemplo, a mi vehículo, fijate que tiene pintura, a mi 4 veces me hicieron atentados. 4 veces. Las 4 veces lo denuncié en la justicia. Nunca la justicia me dio ninguna res- 
puesta. Nunca. Ni me la va a dar. Pero a mí, cuando me judicializan, me tienen todo el día, toda la semana, todo el mes, todo el año, dándome, dándome. Es todo un tema" (Entrevista 12 , con un militante).

La inseguridad no solo se manifiesta en un plano físico y personal, sino también y sobre todo, se refleja en una situación de inestabilidad e inseguridad frente al futuro (Consejo Económico y Social, 1987). La confianza en la accesibilidad y la calidad de los servicios públicos se ha erosionado, sobre todo en el caso de los residentes con menos recursos financieros. Se ha observado que la carencia de una educación de calidad a nivel postsecundario crea descontento. La mayoría de los jóvenes debe ir a vivir lejos para realizarse a través de estudios universitarios, opción que solo es accesible para las familias con medios económicos suficientes. En lo que se refiere al sistema de salud, los testimonios indican carencias importantes en Andalgalá. Para resolver cualquier problema de salud que requiera una atención especializada, hay que viajar a la capital, a la ciudad de Catamarca, lo que representa tres horas y media de transporte, además de importantes costos para las familias.

En cuanto a la situación de la mujer, toda la información recopilada muestra que su situación ha empeorado en las últimas décadas. Diversos entrevistados esgrimen que el número de madres jóvenes solteras es cada vez mayor, lo que aumenta la precariedad en general en la comunidad. A partir de nuestro trabajo de terreno y de las fuentes consultadas podemos concluir que la aplicación del modelo extractivista en Andalgalá ha creado muy pocas oportunidades para la población en general y para las mujeres en particular. En el caso de la población femenina, solamente el $8 \%$ ha podido acceder a puestos en el proyecto Bajo la Alumbrera, todos ellos de naturaleza subalterna (Entrevista 17, con una trabajadora).

\section{La relación con el espacio de vida y el deterioro medioambiental}

La piedra angular del rechazo contra el extractivismo es el medio ambiente, especialmente la contaminación del agua, del aire y del suelo. Los megaproyectos mineros necesitan cantidades desproporcionadas de agua y de electricidad comparativamente con el resto de las actividades. Minera Alumbrera ha construido su propia infraestructura para garantizar la alimentación en agua y electricidad para su proyecto. La empresa consume el equivalente de $170 \%$ de la energía consumida por la provincia de Catamarca en su conjunto y tiene licencia para extraer el equivalente de 100 millones de litros de agua por día (Machado Aráoz, 2009a). Aunque no dependa de las infraestructuras públicas para ello, es seguro que compite con las otras actividades económicas por el uso de los recursos, sobre todo si se tiene en cuenta el clima semidesértico de la provincia de Catamarca.

Los activistas entrevistados temen por la contaminación del medio de vida y por ende, el deterioro de la salud. Muchos expresan nostalgia por la época en que no se ponía en duda la pureza de los recursos hídricos. En efecto, el agua está en el centro de las reivindicaciones y acciones sociales contra los proyectos mineros. Como Machado Aráoz (2009b) lo explica, el desarrollo basado en los grandes proyectos mineros conlleva una manera de entender, vivir y producir el espacio de vida que es incompatible con la aspiración a un buen vivir de la comunidad local. Esto se confirma a través del análisis de las entrevistas realizadas.

Para la industria minera y para los que apoyan el extractivismo, el valor de la tierra se mide por su capacidad para captar inversiones y por las ganancias que pueda producir su explotación. Mientras que, para aquellos que se oponen a dicho modelo, la tierra corresponde al espacio que 
ha sido ocupado por sus ancestros a lo largo de la historia, lo cual ha facilitado el surgimiento de una cultura local específica que se convierte en el sello de la identidad territorial. En la región, tradicionalmente las principales actividades económicas eran la agricultura y la ganadería. Éstas se basaban en una estrecha relación de colaboración entre el ser humano y la naturaleza, mientras que el extractivismo hace que la población local se sienta extranjera en su propio territorio (Machado Aráoz, 2009b).

\section{El declive de los sectores tradicionales}

En cuanto a la riqueza económica como tal, se ha visto que, a juicio de la comunidad, los beneficios económicos a nivel local han sido limitados. Los testimonios recogidos indican que los efectos del proyecto minero se distribuyen de manera desigual a diferentes niveles tanto territorial como social. En lo territorial, se afirma que Andalgalá se ha beneficiado muy poco con las inversiones ligadas a la actividad minera, comparativamente con otros lugares. Y con respecto a lo social, se afirma que solo un grupo social limitado, ligado a la actividad minera como tal, se ha enriquecido mientras que la mayoría de la población vive en una situación precaria.

Si el sector de la minería ha incrementado sus beneficios, otros sectores de la economía se han estancado o han declinado. La agricultura y la industria alimentaria han sido fuertemente afectadas, lo que se ha manifestado en el cierre de muchas fábricas, en la disminución significativa del número de hectáreas explotadas y, por supuesto, en la reducción significativa del empleo disponible en esos sectores. Como se ha visto, después de dos décadas de actividad extractiva, la región muestra una alta tasa de desempleo, la cual puede afectar hasta a un tercio de la población en las zonas rurales de la provincia (Veneranda, 2012). Mientras que la industria minera disfruta de prebendas fiscales otorgadas por el gobierno, las actividades económicas tradicionales no tienen acceso a la misma ayuda. Esto conduce, como dice Canovas (2008), a una desvalorización de las actividades tradicionales por parte de la ideología capitalista.

\section{Conclusión}

Al implantarse en Andalgalá la empresa minera transnacional, se le ha asignado una nueva función a ese territorio en cuanto a la división espacial del trabajo, lo cual ha generado efectos importantes en la colectividad local. El análisis realizado muestra que el desarrollo minero modifica las dinámicas socioterritoriales locales y contribuye al desarrollo geográfico desigual alejando los intereses económicos de las grandes empresas de los intereses de la colectividad. Por otra parte, se acentúa la distinción entre los hombres y las mujeres. En lo que concierne a las mujeres, éstas viven las consecuencias de los proyectos extractivos de manera intensa, lo que se manifiesta en su desvalorización e incide en su reacción, en general también muy intensa. Es interesante destacar que las mujeres sienten que unirse al movimiento antiminero es un deber ya que va de acuerdo a su rol de protección de sus familias y de sus medios de vida.

El proyecto minero ha traído consigo una situación de desarraigo, lo cual tiene efectos en lo que respecta al sentimiento de identidad y de pertenencia (Di Méo, 2004; Lévy \& Lussault, 2013). Bassand y Guindani (1983) han mostrado que este sentimiento puede ser tanto positivo como negativo. Si la identidad es positiva, ella da lugar a "emblemas" que movilizan a los ciudadanos 
residentes en un territorio y que motivan su compromiso con el bien común y el desarrollo colectivo. Si la identidad es negativa actúa como un estigma, lo que se traduce por la desmovilización colectiva. El extractivismo ha provocado una mutación en la identidad de amplias capas de los habitantes de Andalgala, muchos de los cuales se sienten estigmatizados, como lo precisa Cardinal (2009) a un nivel más general. Como Canovas (2008) lo ha mostrado, la difusión de los modelos de desarrollo ligados a la visión neoliberal del territorio modifica negativamente la identidad de muchos residentes, lo que según Klein (2005), altera su compromiso con la colectividad.

Esta mutación identitaria se acentúa por el hecho de que los proyectos mineros tienen una duración limitada, aunque sus efectos sean durables. El promedio de la duración de estos proyectos se sitúa entre 15 y 25 años, es decir que son inferiores a una generación, (Amilhat Szary, 2010). La fase de la extracción de "Bajo la Alumbrera" comenzó en 1997 y la compañía anunció su cese completo para antes del 2018. Pocos empleos locales estables han sido creados a través de este proyecto y cuando los aproximadamente 100 empleados acrecienten las filas de los desempleados, el impacto en la economía local será importante, lo que es fuente de inseguridad y crea un sentimiento de precariedad. Si las cifras de "Minera Alumbrera" son correctas y si es cierto que cada puesto de trabajo en la mina permite mantener a otros cinco trabajadores en la comunidad de residencia, es toda la economía de Andalgalá que se resentirá con el cierre ya anunciado de la mina.

La falta de control local sobre la gran empresa puede ser devastador para las poblaciones locales. Como lo sugiere Maris (2009), las mujeres son las primeras víctimas de un modelo como el extractivismo, y también son las primeras en reaccionar. Es significativo que el organismo "Mujeres del Silencio" denuncie a la empresa minera tanto por sus efectos locales, como en lo que respecta al modelo de sociedad en el cual dicha empresa se inserta (Veneranda, 2012). Es quizás la toma de conciencia de una nueva forma de colonialismo que lleva a las mujeres a luchar contra las políticas extractivas y a proponer una visión alternativa basada en la calidad de vida y, por ende, en la protección del medio de vida, es decir del territorio.

El territorio local permite reconstruir lazos sociales y relaciones de solidaridad (Klein, 2005). Esto, sin embargo, no es automático. La construcción de un modelo alternativo de desarrollo constituye uno de los grandes retos de nuestra época. El territorio es una base a partir de la cual son lanzadas acciones colectivas que despiertan o refuerzan la conciencia territorial. Esto exige una opción territorial que incluya una gobernanza multi-escalar, que incluya la interrelación entre lo local, lo nacional y lo mundial, pero a partir de la base. Es necesario poner en práctica modalidades estratégicas de desarrollo territorial que articulen lo productivo y lo social en una perspectiva de economía social y solidaria (Laville \& Coraggio, 2016). En lo territorial, esto se traduce en una visión que plantee modalidades incluyentes de valorización de los recursos, donde el bienestar de la colectividad prime sobre los intereses financieros. Así planteado, el desafío es elaborar una visión multifacética en la cual lo local permita recrear lo global imbricando las diversas escalas del desarrollo, y en la cual el desarrollo no se haga a expensas de la colectividad sino en su beneficio.

\section{Referencias bibliográficas}

ALPE, Y. \& BEITONE, A. (director). Lexique de sociologie. Paris: Dalloze-Sirey, 2007. 
ALVAREZ, L.M. Los impactos económicos y sociales del proyecto Bajo la Alumbrera y una aproximación a los indicadores económicos de sustentabilidad. Indicadores de Sostenibilidad para la Industria Extractiva Mineral, 2011, p. 319-336.

AMILHAT SZARY, A.-L. Les entreprises minières dans la gouvernance territoriale - Entre négociations de pouvoir et rivalités territoriales, qui porte le développement aux habitants? Revue Géographique de l'Est, 2010, N³-4, p. 1-15.

BEBBINGTON, A.; HUMPHREYS BEBBINGTON, D.; BURY, J., LINGAN J. \& MUÑOZ, J.P. Mining and social movements: struggles over livelihood and rural territorial development in the Andes. World Development, 2008, №12, p. 2888-2905.

BASSAND, M. \& GUINDANI, S. Maldéveloppement régional et luttes identitaires. Espaces et sociétés, $N^{\circ} 42,1983$, p.13-26.

BENKO, G. \& LIPIETZ, A. Les régions qui gagnent. Paris: Presses universitaires de France, 1992.

BOLSI, A.; LONGHI, F. y PAOLASSO, P. Pobreza y mortalidad infantil en el norte grande Argentino. Un aporte para la formulación de políticas públicas. Cuadernos geográficos, 2010, № 45, p. 231-261.

CANOVAS, J. Nouveaux mouvements sociaux et néolibéralisme en Amérique latine. Des alternatives à un système globalisé? Paris: L'Harmattan, 2008.

CARDINAL, A. Savoirs traditionnels et développement : apports critiques. Montréal: Memoria de maestría, Université du Québec à Montréal, 2010.

CASTEL, R. La citoyenneté sociale menacée. Cités, № 135, p. 133-141

CHIASSON-LEBEL, T. Les indicateurs sociaux dans les Amériques : les disparités entre hommes et femmes (1990-2005). Observatoire des Amériques, 2006, p.1-39.

COMELLI, M. Autoconvocadas por la vida. Mujeres en acción frente a la megaminería a cielo abierto en Tinogasta, Catamarca. Conflicto Social, 2010, № 3, Disponible en Internet:

http://www.ger-gemsal.org.ar/wp-content/imagenes/Comelli-Autoconvocadas-por-la-vida.pdf

CONSEIL ECONOMIQUE ET SOCIAL. Grande pauvreté et précarité économique et sociale. Paris: République française, rapport des séances des 10 et 11 février 1987.

CORIA, L.G. Desarrollo local y actividad minera en la provincia de Catamarca. Revista Oidles, 2007, No 1, p.1-38.

DABENE, O. (director). La gauche en Amérique latine. Paris: Presses de la fondation nationale des sciences politiques, 2012.

DE MATTOS, C.; FIGUEROA, O.; GIMENEZ I CAPDEVILA, R.; ORELLANA, A. y G.YÁÑEZ WARNER (directores). Gobernanza, competitividad y redes. La gestión de las ciudades en el siglo XXI. Santiago de Chile: EURE Libros, Colección RIDEAL, 2005. 
DI MEO, G. Les paysages de l'identité. Géocarrefour, 2004, N² 2, p. 131-141.

DIRECCIÓN NACIONAL DE RELACIONES ECONÓMICAS CON LAS PROVINCIAS. Informes Sintéticos de Caracterización Socio-Productiva. Catamarca, 2012.

GORENSTEIN, S. Transformaciones territoriales contemporáneas. Desafíos del pensamiento latinoamericano. EURE, 2015, Vol. 41, No 122, p. 5-26.

GUDYNAS, E. Geografías fragmentadas: sitios globalizados, áreas relegadas. Revista del Sur, 2005, No 160, p. 3-13.

GUDYNAS, E. Conflictos ambientales en zonas de frontera y gestión ambiental en América del Sur. Gestión Ambiental, 2007, №13, p. 1-19.

HARTSOCK, N. Globalization and Primitive Accumulation: The Contributions of David Harvey's Dialectical Marxism. In: CASTREE, N. \& GREGORY, D. David Harvey: A Critical Reader, Blackwell Publishing, 2008, p. 167-190.

HARVEY, D. Géographie et capital. Vers un matérialisme historico-géographique. Paris: Éditions Syllepses, 2010.

KLEIN, J.-L. Iniciativa local y desarrollo. EURE, 2005, Vol. 31, N94, p. 25-39.

KLEIN, J.-L. Territoire et régulation. Cahiers de recherche sociologique, 2008, № 45, p. 41-58.

KLEIN, J.-L. L'espace local à l'heure de la globalisation: la part de la mobilisation sociale. Cahiers de géographie du Québec, Vol. 41, Nº 114, 1997, p. 367-377.

KLEIN, J.-L. Économie sociale et territoire en contexte de mondialisation : le développement par I'initiative locale. In : BELLEMARE, G. \& KLEIN, J-L. Innovation sociale et territoire Convergence théoriques et pratiques. Québec: Presses de I'Université du Québec, 2011.

KLEIN, J.-L.; FONTAN, J.-M. \& D.-G. TREMBLAY. Social Entrepreneurs, Local Initiatives and Social Economy: Foundations for a Socially Innovative Strategy to Fight against Poverty and Exclusion. Canadian Journal of Regional Research/Revue canadienne de science régionale. Vol. 32, N. 1, 2009, p. 23-42.

KLEIN, J.-L. \& R. GUILLAUME (directores). Vers une nouvelle géographie économique. Québec: Presses de l'Université du Québec, 2014.

KLEIN, J.-L. \& LAURIN, S. (directores). L'Éducation géographique. Conscience territoriale et formation du citoyen. Sainte-Foy: Presses de l'Université du Québec, 1999.

LACOMBE, M. L'action collective locale face à l'agroindustrie : le cas du movimiento campesino de Córdoba dans la province de Córdoba en Argentine. Montréal: Cahiers du CRISES, TM1201, 2012. 
LAMALICE, A. Extractivisme et développement inégal, le cas de l'industrie minière dans la province de Catamarca en Argentine. Montreal: Tesis de maestría en Geografía, Universidad de Quebec en Montreal, 2014.

LAVILLE, J.L. \& CORAGGIO, J.L. (directores). Les gauches du XXI siècle. Lormont: Editions Le bord de l'eau, 2016

LEBLANC, J. La nécessité d'une perspective écoféministe politique. 2010. Disponible en Internet: http://www.moutonnoir.com/2010/03/la-necessited\%e2\%80\%99une-perspective-ecofeministe-politique/

LÉVY, J. \& LUSSAULT, M. Dictionnaire de la géographie et de l'espace des sociétés. Paris: Belin, 2013.

LIPIETZ, A. Le fordisme périphérique étranglé par le monétarisme. L'Actualité économique, 1984, $N^{\circ} 1$, p. $72-94$.

MACHADO ARÁOZ, H. Minería transnacional, conflictos socioterritoriales y nuevas dinámicas expropiatorias. El caso de Minera Alumbrera. En: SVAMPA, M. y ANTONELLI, M.A. (editores). Mineria transnacional, narrativas del desarrollo y resistencias sociales. Buenos Aires: Editorial Biblos Sociedad, 2009a, p. 205-228.

MACHADO ARÁOZ, H. Identidades en conflicto. Reconversión neocolonial, Conflictos socioterritoriales y procesos de subjetivación en un contexto periférico del capitalismo global. Boletín Onteaiken, 2009b, №7, p. 73-87.

MAGRIN, G. \& PERRIER-BRUSLÉ, L. Nouvelles géographies des activités extractives. Echogeo, 2011. Disponible en Internet : http://echogeo.revues.org/12586

MARIS, V. Quelques pistes pour un dialogue fécond entre féminisme et écologie. Multitudes, 2009, N³6, p. 178-184.

MARTINEZ ALIER, J. Mining conflicts, environmental justice, and valuation. Journal of Hazardous Materials, 2006, № 86, p.153-170.

MINERA ALUMBRERA. Características generales. 2004. Disponible en Internet: http://www.alumbrera.com.ar/institucional.asp

MINERA ALUMBRERA. Informe de Sostenibilidad 2012. Minera Alumbrera YMAD-UTE \& Xstrata copper. Disponible en Internet: http://www.alumbrera.com.ar

MINGIONE, E. L'innovation sociale face aux défis de la globalisation. Tensions et illusions. In : KLEIN, J.-L. : CAMUS, A.; JETTÉ, C.; CHAMPAGNE, CH. \& M. ROY (directores). La transformation sociale par l'innovation sociale. Québec: Presses de l'Université du Québec, 2016, p. 35-47. 
NATERA RIVAS, J.J. Caracterización de la pobreza según el nivel de renta en el noroeste argentino. Ería, 2005, №67, p. 193-208.

ORELLANA, R. Aproximaciones a un marco teórico para la comprensión y el manejo de conflictos socioambientales. En: ORTIZ, T. Comunidades y ConflictosSocioambientales: experiencias y desafiaos en América Latina. Quito: Ediciones Abya-Yala, 1999, p.89-108.

ROSE, D. \& GILBERT, A. Glimpses of social and cultural geography in Canada and Quebec at the turn of the millennium. Social \& Cultural Geography, 2005, № 2, p. 271-293.

SASSEN, S. A Sociology of Globalization. New York: W.W. Norton, 2007.

SCOTT, A. (director). Global City-Regions. Trends, Theory, Policy. Oxford: Oxford University Press, 2001.

SECRETARIA DE MINERÍA DE LA NACIÓN. Minería en números. 2013. Disponible en Internet: http:// www.mineria.gob.ar/

SOUSSI, S.A. \& BEAUDET, P. Du prolétariat au précariat: introduction au dossier. Nouveaux Cahiers du Socialisme, 2012, No 7, p 9-12.

SVAMPA, M. Néo-"développementisme" extractiviste, gouvernements et mouvements sociaux en Amérique latine. Problèmes d'Amérique latine, 2011, № 81, p. 103-127.

SVAMPA, M.; BOTTARO, L. y SOLA ALVAREZ, M. La problemática de la minería metalífera a cielo abierto: modelo de desarrollo, territorio y discursos dominantes. En: SVAMPA, M. y ANTONELLI, M.A. (editores). Mineria transnacional, narrativas del desarrollo y resistencias sociales. Buenos Aires: Editorial Biblos Sociedad, 2009a, p.29-50.

VELUT, S. Argentine - Chili : Une si longue frontière. Confins-Revue Franco-brésilienne de géographie, 2009, $N^{\circ}$ 7. Disponible en Internet: https://confins.revues.org/6095\#quotation

VENERANDA, M. Las mujeres del silencio avivan la guerra a la minería. Diario La Nación. 2012. Disponible en Internet: http://www.lanacion.com.ar/1449810-las-mujeres-del-silencio-avivan-laguerra-a-la-mineria

WALTER, M. Conflictos ambientales, socioambientales, ecológico distributivos, de contenido ambiental... Reflexionando sobre enfoques y definiciones. Boletín ECOS, 2009, $N^{\circ} 6$. Disponible en Internet:

http://www.fuhem.es/media/ecosocial/file/Boletin\%20ECOS/Boletin\%206/Conflictos\%20ambientales_m.walter_mar09_final.pdf 
